anti-inflammatory effects and confer predisposition to gout, with additional evidence suggesting that recombinant IL-37 could have therapeutic potential in the disease. "This is a completely new finding in the field of gout and IL-37 biology," notes corresponding author Leo Joosten.

IL-1 $\beta$-mediated inflammation is central to the pathogenesis of gout. The IL- 1 family member IL-37 is known to be a negative regulator of IL- $1 \beta$ signalling, and its anti-inflammatory properties have been demonstrated in numerous disease models. However, the contribution of IL-37 to the disease process in gout was unclear.

In the present study, the researchers identified four distinct rare variants of $I L 37$ in six patients with gout (within the discovery molecular inversion probe-based resequencing technology. The four variants were clustered in exon 5 , which encodes the functional domain of IL-37. None of the variants was found in a control cohort of 520 healthy adults.

The researchers also observed that the patients who were carriers of the rare IL37 variants had either onset of gout at a younger age or a more severe disease phenotype, with multiple inflammatory comorbidities, compared with noncarriers. Predictive modelling and in vitro studies confirmed that the variants affect the structure of the IL-37 protein and consequently its anti-inflammatory function.

In monosodium urate (MSU)stimulated polymorphonuclear cells from a patient carrying one of the four rare variants, treatment administration of recombinant IL-37 dampened the inflammation induced by MSU crystals

\title{
Proteinase KLK6 links skin and joint inflammation in PsA
}

Individuals with psoriasis have an increased risk of developing psoriatic arthritis ( $\mathrm{PsA})$, but the exact mechanisms that link skin inflammation and joint disease are unclear. The results of a new study suggest that in mice, signalling between the serine proteinase kallikrein-related peptidase 6 (KLK6) and proteinase-activated receptor 1 (PAR1) in the skin alone might be sufficient to trigger joint disease.

"The idea that overexpressing a serine protease in the epidermis could be sufficient to cause damage to the joints and bones at distant sites was what got us really excited," enthuses corresponding author Nicole Ward. "Preclinical reports have shown that skin-initiated inflammation can lead to arthritis-like changes, so our findings support the idea that chronic inflammation in the skin has the

capacity to promote distant damage to the joints and bone, and that

the skin inflammation

was necessary

for the arthritis to persist

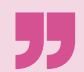
eliminating the skin inflammation can reduce that damage."

To investigate the role of KLK6 in psoriasis and PsA, Ward and colleagues developed transgenic mice with overexpression of Klk6 in their keratinocytes. This overexpression of Klk6 could be switched off by the administration of doxycycline. These mice, known as Klk6 $6^{+}$ transgenic mice, developed dermatitis that histologically and transcriptionally mirrored human psoriatic skin lesions. Interestingly, the mice also spontaneously developed dactylitis, enthesitis, synovitis, kyphosis and had reduced vertebral bone mineral density, suggestive of a disease similar to that seen in patients with PsA.

Reduction of the Klk6 expression in these mice to wild-type levels with with recombinant IL-37 reduced the production of IL- 8 and reactive oxygen species. Moreover, in a mouse model of arthritis, administration of recombinant IL-37 dampened the inflammation induced by MSU crystals in wild-type mice.

"We are planning to perform experiments to treat mice with an IL-37 fusion protein," says Joosten. If those experiments prove successful, the researchers plan to undertake clinical studies to evaluate the treatment in patients with gout.

Sarah Onuora

ORIGINAL ARTICLE Klück, V. et al. Rare genetic variants in interleukin-37 link this antiinflammatory cytokine to the pathogenesis and treatment of gout. Ann. Rheum. Dis. https://doi.org/10.1136/ annrheumdis-2019-216233 (2020) doxycycline ameliorated the skin inflammation and caused a reversal of at least some of their joint disease, suggesting that the skin inflammation was necessary for the arthritis to persist. Knockout experiments revealed a role for PAR1, but not for PAR2, in KLK6 signalling, and follow-up experiments on human psoriatic skin explants showed a reduction in the production of pro-inflammatory cytokines upon treatment with the PAR1 inhibitor vorapaxar.

"We are focused on taking this work forward by using the $K l k 6^{+}$transgenic mice to study the mechanisms of action by which skin inflammation causes arthritis-like damage and by translating these findings back into mechanisms that are relevant to patients with PsA," concludes Ward.

\section{Joanna Clarke}

ORIGINAL ARTICLE Billi, A. C. et al. KLK6 expression in skin induces PAR1-mediated psoriasiform dermatitis and inflammatory joint disease.J. Clin. Invest. https://doi.org/10.1172/ JCl133159 (2020)

RELATED ARTICLE Oikonomopoulou, K. et al. Proteinases and their receptors in inflammatory arthritis: an overview. Nat. Rev. Rheumatol. 14, 170-180 (2018) 